\title{
Automated Facilitation Support in Online Forum
}

\author{
Wen Gu* \\ Nagoya Institute of Technology \\ University of Wollongong \\ gu@ katolab.nitech.ac.jp
}

\begin{abstract}
Online forum that gathers participants together to solve the common issues that they are facing is considered as a promising application of utilizing collective intelligence to solve complicated realworld problems. To facilitate the discussions in online forum to proceed smoothly and to build consensus efficiently, human facilitators are introduced into the system. With the increasing sophistication of online forum, human facilitators related problems such as human bias and restricted scale become critical. Therefore, it is critical to explore approaches to support human facilitators in conducting facilitation. However, most of the existing facilitation support techniques only support predefined facilitation tasks that could be defined by static rules. In this research, we aim to explore potential solutions for supporting the human facilitators to conduct facilitation in online forum. As the first step, we have proposed a case-based reasoning (CBR)-based framework that targets support facilitation by utilizing past successful facilitation experience. Currently, our work is focusing on the specific facilitation task of detecting influential user in the online forum. In the future work, we are planning to propose approaches of solving other specific facilitation tasks such as measuring the level of agreement and encouraging participants to reach a consensus.
\end{abstract}

\section{Introduction}

With the development of the Internet, online forum has made it possible to gather collective intelligence to solve critical social problems with less restriction such as time issue and location issue. It has been practically used in the situations of global climate change, city planning and urban dialogue [Ito et al., 2014] [Haqbeen et al., 2020]. In the online forum, human facilitators are introduced in order to facilitate the discussion to proceed smoothly and to achieve consensus efficiently. However, due to the lack of common definition of online forum facilitation, human bias becomes inevitable for

\footnotetext{
${ }^{*}$ Contact Author
}

human facilitators. Also, it is difficult for human facilitators to handle the increasing participant scale. Therefore, supporting human facilitators becomes a critical issue for the online forum.

In my doctoral research, I focus on developing automated online forum facilitation support technique that could provide answers in solving these above mentioned issues. First, we consider to support human facilitators by utilizing past successful facilitation experience. The particular reason of this idea is that experience accumulation is the key factor for a novice human facilitator to become an experienced human facilitator. In addition, well formalized experience could be utilized as standard facilitation reference. As a result, we propose a CBR-based facilitation support approach that aims to accumulate and reuse the general successful facilitation experience [Gu et al., 2021]. Facilitated online discussion is formally defined and the process of building case base, retrieving similar case are elaborated. During this research, we realize that facilitation in online forum is a complicated issue that contains various of sub-tasks. To pursue more precisely support for the human facilitators, these detailed sub-tasks should be resolved. Presently, we are working on the influential user detection which is a vital task for group analysis and group decision making. A number of facilitation strategies such as contents generation and participation encouragement are determined with the understanding of the influential participants. In particular, we propose to build an online forum social network based on the post reply relation between users because we argue that influence propagates in the online forum when one participant replies to another participant. Other specific facilitation tasks such as measuring the level of agreement and encouraging participants to reach a consensus are also considered as the future directions.

\section{Completed Research}

As the first step of my research, we have proposed a casebased reasoning (CBR)-based framework to support human facilitators in conducting facilitation. CBR is selected because it possesses similar reasoning paradigm as experienced human facilitators, which leverages the accumulation of successful experience to tackle new facilitation problem. CBR provides an effective reasoning paradigm for solving new problems by adopting similar solutions that have been proposed for similar problems in the past [Schank, 1983]. In 
the proposed framework, the research problem of facilitation support in online forums is formally defined by four main components. First, defining a discussion case and building a case base that collects various online forum cases are the base of the framework. Each case should contain one case description part that stores online forum situation and one case solution part that stores generated facilitation. In particular, we modeled the case description, i.e., online forum, in issuebased information system (IBIS) style [Conklin, 2005]. As an argumentation approach to clarify complex problems that involve multiple stakeholders, IBIS is utilized to extract the information that human facilitators use in their decision making in order to build a computational model. Second, similarity between cases are calculated to retrieve the most similar case. Specifically, similarity is calculated based on the idea of comparing the number of features which are common to both graphs to the total number of features. After the case retrieval, the case solution part in the retrieved case is reused as the facilitation suggestion for the human facilitators and case retain is determined after verification. In this process, the knowledge of online forum facilitation is accumulated in the system.

To evaluate the proposed framework, we built an online discussion case base based on a set of annotated real-world discussion data and conducted a set of experiments. Generated facilitation support information by the proposed framework is validated to be useful from the experimental results.

\section{Ongoing Work}

Currently, my work targets one of the critical facilitation tasks in online forum, i.e., influential user detection, which could be utilized as the reference of generating facilitation strategy. Specifically, I focus on detecting the influential user from the influence propagation perspective. Detecting influential user is considered as one of the most valuable research problems in the research domain of online social network analysis. In this research area, most work define online social network as a graph where users are defined as nodes and connected with each other by beforehand known relationships or interactions [Li et al., 2018]. Influence user detection in this area is well known as the influence maximization (IM) problem, which aims to select a subset of $k$ users, i.e., seed set, from the whole social network user set [Kempe et al., 2003]. However, conventional social network generation that utilizes existing friend relations or follower relations is not applicable in online forum. In addition, the characteristics of users and influence propagation in online forum cannot not be reflected by conventional social network.

To this end, I propose to build an online forum influence propagation network on the basis of the post reply relation between users, which is regarded as the sign of influence propagation from one user to another user in the online forum. Based on this built influence propagation network, it becomes possible to simulate the influence propagation from one participant to another participant in the online forum. The characteristics of each participant are introduced in order to represent the differences of online forum participants. In specific, one of the introduced factors is user contribution, which represents individual contribution to the development of the discussion. Another factor is user relevance, which represents the communication degree between two users. Influential users are detected from the network based on the introduced characteristics and validated by influence propagation simulation. As a result, detected influential users will be utilized as facilitation strategy reference by human facilitators.

\section{Future Work}

In my doctoral research, my focus lies in the facilitation support in online forum. I have already proposed a framework based on the experienced human facilitators thinking paradigm and currently working on the topic of influential users detection from the online forum. I aim to finish the current work and analyze the influence propagation in different online forum situations in my doctoral research. From the current research results, the complexity of the facilitation in the online forum and the necessity of investigating the facilitation tasks in detail are all valuable insights that I have understood. As a result, further research attention should be paid to these correlated tasks. For instance, designing a computational model to calculate the level of agreement or the level of satisfaction are challenging tasks. Evaluation of the generated facilitation support in the real world online forum is also critical for this research.

\section{References}

[Conklin, 2005] Jeff Conklin. Dialogue Mapping: Building Shared Understanding of Wicked Problems. John Wiley \& Sons, Inc., New York, NY, USA, 2005.

[Gu et al., 2021] Wen Gu, Ahmed Moustafa, Takayuki Ito, Minjie Zhang, and Chunsheng Yang. A case-based reasoning approach for supporting facilitation in online discussions. Group Decis Negot 30, pages 719-742, 2021.

[Haqbeen et al., 2020] Jawad Haqbeen, Takayuki Ito, Rafik Hadfi, Tomohiro Nishida, Zoia Sahab, Sofia Sahab, Shafiq Roghmal, and Ramin Amiryar. Promoting discussion with ai-based facilitation: Urban dialogue with kabul city. In proceeding of the 8th ACM Collective Intelligence Conference, 2020.

[Ito et al., 2014] Takayuki. Ito, Yuma. Imi, Takanori Ito, and Eizo. Hideshima. Collagree: A faciliator-mediated largescale consensus support system. In Collective Intelligence. MIT Cambridge, USA, 2014.

[Kempe et al., 2003] David Kempe, Jon Kleinberg, and Éva Tardos. Maximizing the spread of influence through a social network. In Proceedings of the ninth ACM SIGKDD international conference on Knowledge discovery and data mining, pages 137-146, 2003.

[Li et al., 2018] Yuchen Li, Ju Fan, Yanhao Wang, and KianLee Tan. Influence maximization on social graphs: A survey. IEEE Transactions on Knowledge and Data Engineering, 30(10):1852-1872, 2018.

[Schank, 1983] Roger C. Schank. Dynamic Memory: A Theory of Reminding and Learning in Computers and People. Cambridge University Press, New York, NY, USA, 1983. 